\title{
Unification of Electromagnetic Interactions and Gravitational Interactions
}

\author{
Ning $\mathrm{Wu}^{*}$ \\ Institute of High Energy Physics, P.O.Box 918-1, Beijing 100039, P.R.China ${ }^{\dagger}$
}

November 1, 2018

PACS Numbers: 04.50.+h, 12.10.-g, 04.60.-m, 11.15.-q.

Keywords: quantum gravity, unified field theory, gauge field theory.

\begin{abstract}
Unified theory of gravitational interactions and electromagnetic interactions is discussed in this paper. Based on gauge principle, electromagnetic interactions and gravitational interactions are formulated in the same manner and are unified in a semi-direct product group of $U(1)$ Abel gauge group and gravitational gauge group.
\end{abstract}

*email address: wuning@mail.ihep.ac.cn

${ }^{\dagger}$ mailing address 


\section{Introduction}

It is known that there are four kinds of fundamental interactions in Nature, which are strong interactions, electromagnetic interactions, weak interactions and gravitational interactions. All these fundamental interactions can be described by gauge field theories, which can be regarded as the common nature of all these fundamental interactions. This provides us the possibilities to unify different kinds of fundamental interactions in the framework of gauge theory. The first unification of fundamental interactions in human history is the unification of electric interactions and magnetic interactions, which is made by Maxwell in 1864. Now, we know that electromagnetic theory is a $U(1)$ Able gauge theory. In 1921, H.Weyl tried to unify electromagnetic interactions and gravitational interactions in a theory which has local scale invariance[1,2]. Weyl's original work is not successful, however in his great attempt, he introduced one of the most important concept in modern physics: gauge transformation and gauge symmetry. After the foundation of quantum mechanics, V.Fock, H.Weyl and W.Pauli found that quantum electrodynamics is a $U(1)$ gauge invariant theory $[3,4,5]$.

Gauge treatment of gravity was suggested immediately after the gauge theory birth itself $[6,7,8]$. In the traditional gauge treatment of gravity, Lorentz group is localized, and the gravitational field is not represented by gauge potential $[9,10,11]$. It is represented by metric field. The theory has beautiful mathematical forms, but up to now, its renormalizability is not proved. In other words, it is conventionally considered to be non-renormalizable. Recently, some new attempts were proposed to use Yang-Mills theory to reformulate quantum gravity[12, 13, 14, 15]. In these new approaches, the importance of gauge fields is emphasized. Some physicists also try to use gauge potential to represent gravitational field, some suggest that we should pay more attention on translation group. Recently, Wu proposed a new quantum gauge theory of gravity which is a renormalizable quantum gravity[16, 17]. Based on gauge principle, space-time translation group is selected to be the gravitational gauge group. After localization of gravitational gauge group, the gravitational field appears as the corresponding gauge potential. In this paper, we use the spirit inspired by literature $[16,17]$ to discuss unification of fundamental interactions which is based on gravitational gauge theory. Our main goal in this paper is to discuss unification of gravitational interactions and electromagnetic interactions. 


\section{Lagrangian}

It is known that Quantum Electrodynamics(QED) is a $U(1)$ Able gauge field theory. A general group element of $U(1)$ Able gauge group is denoted as $U(x)$,

$$
U(x)=e^{-i \alpha(x)} .
$$

A general element of gravitational gauge group is denoted as $\hat{U}_{\epsilon}[16,17]$

$$
\hat{U}_{\epsilon}=E^{-i \epsilon^{\mu} \cdot \hat{P}_{\mu}} \text {. }
$$

Because

$$
\left(\hat{P}_{\mu} \alpha(x)\right) \neq 0,
$$

$U(1)$ group element $U(x)$ does not commute with gravitational gauge group element $\hat{U}_{\epsilon}$,

$$
\left[U(x), \hat{U}_{\epsilon}\right] \neq 0 .
$$

It means that all elements $g(x)$

$$
g(x)=\hat{U}_{\epsilon} \cdot U(x)
$$

form a semi-direct product group of $U(1)$ Able group and gravitational gauge group. We denote it as

$$
G U(1) \triangleq U(1) \otimes_{s} \text { Gravitational Gauge Group }=\{g(x)\} .
$$

This semi-direct product group is the symmetry group of the unified theory of gravitational interactions and electromagnetic interactions.

As an example, we discuss electromagnetic interactions and gravitational interactions between Dirac field and electromagnetic field or gravitational field. The traditional lagrangian density for electromagnetic interactions between electromagnetic field $A_{\mu}$ and Dirac field $\psi$ is given by

$$
-\frac{1}{4} \eta^{\mu \rho} \eta^{\nu \sigma} A_{\mu \nu} A_{\rho \sigma}-\bar{\psi}\left(\gamma^{\mu}\left(\partial_{\mu}-i e A_{\mu}\right)+m\right) \psi
$$

where $A_{\mu \nu}$ is the field strength of electromagnetic field, $e$ is the coupling constant of electromagnetic interactions and $m$ is the mass of Dirac field. After considering gravitational interactions, $A_{\mu \nu}$ is defined by

$$
A_{\mu \nu}=\left(D_{\mu} A_{\nu}\right)-\left(D_{\nu} A_{\mu}\right) .
$$

In above relation, $D_{\mu}$ is the gravitational gauge covariant derivative,

$$
D_{\mu}=\partial_{\mu}-i g C_{\mu}(x),
$$


where $g$ is the gravitational coupling constant and $C_{\mu}(x)$ is the gravitational gauge field which is a vector in gauge group space[16, 17],

$$
C_{\mu}(x)=C_{\mu}^{\alpha}(x) \hat{P}_{\alpha}
$$

The explicit expression for $A_{\mu \nu}$ is

$$
A_{\mu \nu}=\partial_{\mu} A_{\nu}-\partial_{\nu} A_{\mu}-g C_{\mu}^{\alpha} \partial_{\alpha} A_{\nu}+g C_{\nu}^{\alpha} \partial_{\alpha} A_{\mu}
$$

However, $A_{\mu \nu}$ is not a $U(1)$ gauge invariant field strength. In order to define $U(1)$ gauge invariant field strength, we need a matrix $G$. Define,

$$
G \triangleq\left(G_{\mu}^{\alpha}\right) \triangleq\left(\delta_{\mu}^{\alpha}-g C_{\mu}^{\alpha}\right)
$$

Its inverse matrix is denoted as $G^{-1}$,

$$
G^{-1}=\frac{1}{I-g C}=\left(G_{\alpha}^{-1 \mu}\right)
$$

They satisfy the following relations,

$$
\begin{aligned}
& G_{\mu}^{\alpha} G_{\alpha}^{-1 \nu}=\delta_{\mu}^{\nu}, \\
& G_{\beta}^{-1 \mu} G_{\mu}^{\alpha}=\delta_{\beta}^{\alpha} .
\end{aligned}
$$

It can be proved that

$$
D_{\mu}=G_{\mu}^{\alpha} \partial_{\alpha}
$$

The field strength of gravitational gauge field is defined by

$$
F_{\mu \nu} \triangleq \frac{1}{-i g}\left[D_{\mu} \quad, \quad D_{\nu}\right]
$$

Its explicit expression is

$$
F_{\mu \nu}(x)=\partial_{\mu} C_{\nu}(x)-\partial_{\nu} C_{\mu}(x)-i g C_{\mu}(x) C_{\nu}(x)+i g C_{\nu}(x) C_{\mu}(x) .
$$

$F_{\mu \nu}$ is also a vector in gauge group space,

$$
F_{\mu \nu}(x)=F_{\mu \nu}^{\alpha}(x) \cdot \hat{P}_{\alpha},
$$

where

$$
F_{\mu \nu}^{\alpha}=\partial_{\mu} C_{\nu}^{\alpha}-\partial_{\nu} C_{\mu}^{\alpha}-g C_{\mu}^{\beta} \partial_{\beta} C_{\nu}^{\alpha}+g C_{\nu}^{\beta} \partial_{\beta} C_{\mu}^{\alpha} .
$$

The $U(1)$ gauge invariant field strength of electromagnetic field is given by the following relation,

$$
\mathbb{A}_{\mu \nu}=A_{\mu \nu}+g G_{\alpha}^{-1 \lambda} A_{\lambda} F_{\mu \nu}^{\alpha}
$$


where $A_{\mu \nu}$ is defined by eq.(2.8). $G U(1)$ gauge covariant derivative is

$$
\mathbb{D}_{\mu}=\partial_{\mu}-i e A_{\mu}-i g C_{\mu}
$$

The gravitational gauge covariant and $U(1)$ gauge invariant lagrangian density $\mathcal{L}_{0}$ is

$$
\mathcal{L}_{0}=-\bar{\psi}\left(\gamma^{\mu} \mathbb{D}_{\mu}+m\right) \psi-\frac{1}{4} \eta^{\mu \rho} \eta^{\nu \sigma} \mathbb{A}_{\mu \nu} \mathbb{A}_{\rho \sigma}-\frac{1}{4} \eta^{\mu \rho} \eta^{\nu \sigma} \eta_{2 \alpha \beta} F_{\mu \nu}^{\alpha} F_{\rho \sigma}^{\beta},
$$

where $\eta^{\mu \nu}$ is the Minkowski metric. The selection of $\eta_{2 \alpha \beta}$ is not unique[16, 17]. One selection is to set

$$
\eta_{2 \alpha \beta}=\left(\begin{array}{cccc}
-1 & 0 & 0 & 0 \\
0 & 1 & 0 & 0 \\
0 & 0 & 1 & 0 \\
0 & 0 & 0 & 1
\end{array}\right)
$$

Another selection is to set

$$
\eta_{2 \alpha \beta}=g_{\alpha \beta}
$$

where

$$
g_{\alpha \beta} \triangleq \eta_{\mu \nu}\left(G^{-1}\right)_{\alpha}^{\mu}\left(G^{-1}\right)_{\beta}^{\nu}
$$

The first choice gives out minimum model, so we use the first choice in this paper.

The full lagrangian density $\mathcal{L}$ is defined by

$$
\mathcal{L}=J(C) \mathcal{L}_{0}
$$

where $J(C)$ is a special factor to resume gravitational gauge symmetry of the system. The selection of $J(C)$ is not unique. The simplest and most beautiful choice of $J(C)$ is $[16,17]$,

$$
J(C)=e^{I(C)},
$$

where

$$
I(C)=g \eta_{1 \alpha}^{\mu} C_{\mu}^{\alpha} .
$$

The definition of $\eta_{1 \alpha}^{\mu}$ can be found in Ref.[16, 17]. Another possible definition of $J(C)$ is

$$
J(C)=\sqrt{-\operatorname{det} g_{\alpha \beta}}
$$

where $g_{\alpha \beta}$ is given by eq.(2.26) In this paper, we will use definition eq.(2.28), for this is the simplest and most beautiful choice which has no poles in it. The action of the system is

$$
S=\int \mathrm{d}^{4} x \mathcal{L}
$$




\section{Gauge Symmetry}

Now, let's study the symmetry of the system. First, let's discuss $U(1)$ gauge symmetry of the system. Under local $U(1)$ gauge transformations, the transformations of various fields are

$$
\begin{gathered}
\psi \rightarrow \psi^{\prime}=e^{-i \alpha} \psi, \\
C_{\mu} \rightarrow C_{\mu}^{\prime}=C_{\mu}, \\
C_{\mu}^{\alpha} \rightarrow C_{\mu}^{\prime \alpha}=C_{\mu}^{\alpha}, \\
A_{\mu} \rightarrow A_{\mu}^{\prime}=A_{\mu}-\frac{1}{e}\left(D_{\mu} \alpha(x)\right), \\
\mathbb{D}_{\mu} \rightarrow \mathbb{D}_{\mu}^{\prime}=e^{-i \alpha} \mathbb{D}_{\mu} e^{i \alpha}, \\
A_{\mu \nu} \rightarrow A_{\mu \nu}^{\prime}=A_{\mu \nu}+\frac{g}{e} F_{\mu \nu}^{\sigma}\left(\partial_{\sigma} \alpha(x)\right), \\
F_{\mu \nu}^{\alpha} \rightarrow F_{\mu \nu}^{\prime \alpha}=F_{\mu \nu}^{\alpha}, \\
\mathbb{A}_{\mu \nu} \rightarrow \mathbb{A}_{\mu \nu}^{\prime}=\mathbb{A}_{\mu \nu} .
\end{gathered}
$$

From above transformation relations, we could see that gravitational gauge field keeps unchanged under $U(1)$ Abel gauge transformations, and $A_{\mu \nu}$ is not a $U(1)$ gauge invariant field strength while $\mathbb{A}_{\mu \nu}$ is a $U(1)$ gauge invariant. Under $U(1)$ gauge transformation, lagrangian density $\mathcal{L}_{0}$ is invariant,

$$
\mathcal{L}_{0} \rightarrow \mathcal{L}_{0}^{\prime}=\mathcal{L}_{0}
$$

Because $J(C)$ is also $U(1)$ gauge invariant, the full lagrangian density $\mathcal{L}$ and action $S$ of the system are also $U(1)$ gauge invariant,

$$
\begin{gathered}
\mathcal{L} \rightarrow \mathcal{L}^{\prime}=\mathcal{L}, \\
S \rightarrow S^{\prime}=S .
\end{gathered}
$$

Therefore, the system has local $U(1)$ gauge symmetry.

Under gravitational gauge transformations, various fields and operators transform as

$$
\begin{gathered}
\psi \rightarrow \psi^{\prime}=\left(\hat{U}_{\epsilon} \psi\right), \\
C_{\mu} \rightarrow C_{\mu}^{\prime}=\hat{U}_{\epsilon} C_{\mu} \hat{U}_{\epsilon}^{-1}-\frac{1}{i g} \hat{U}_{\epsilon}\left(\partial_{\mu} \hat{U}_{\epsilon}^{-1}\right), \\
G_{\mu}^{\alpha} \rightarrow G_{\mu}^{\alpha}=\Lambda_{\beta}^{\alpha} \hat{U}_{\epsilon} G_{\mu}^{\beta} \hat{U}_{\epsilon}^{-1} \\
G_{\alpha}^{-1 \mu} \rightarrow G_{\alpha}^{\prime-1 \mu}=\Lambda_{\alpha}{ }^{\beta} \hat{U}_{\epsilon} G_{\beta}^{-1 \mu} \hat{U}_{\epsilon}^{-1}
\end{gathered}
$$




$$
\begin{gathered}
g_{\alpha \beta} \rightarrow g_{\alpha \beta}^{\prime}=\Lambda_{\alpha}^{\alpha_{1}} \Lambda_{\beta}^{\beta_{1}} \hat{U}_{\epsilon} g_{\alpha \beta} \hat{U}_{\epsilon}^{-1}, \\
A_{\mu} \rightarrow A_{\mu}^{\prime}=\hat{U}_{\epsilon} A_{\mu} \hat{U}_{\epsilon}^{-1}, \\
\mathbb{D}_{\mu} \rightarrow \mathbb{D}_{\mu}^{\prime}=\hat{U}_{\epsilon} \mathbb{D}_{\mu} \hat{U}_{\epsilon}^{-1}, \\
A_{\mu \nu} \rightarrow A_{\mu \nu}^{\prime}=\hat{U}_{\epsilon} A_{\mu \nu} \hat{U}_{\epsilon}^{-1}, \\
F_{\mu \nu}^{\alpha} \rightarrow F_{\mu \nu}^{\alpha}=\Lambda_{\beta}^{\alpha} \hat{U}_{\epsilon} F_{\mu \nu}^{\beta} \hat{U}_{\epsilon}^{-1}, \\
\mathbb{A}_{\mu \nu} \rightarrow \mathbb{A}_{\mu \nu}^{\prime}=\hat{U}_{\epsilon} \mathbb{A}_{\mu \nu} \hat{U}_{\epsilon}^{-1}, \\
J(C) \rightarrow J^{\prime}\left(C^{\prime}\right)=J \cdot \hat{U}_{\epsilon} J(C) \hat{U}_{\epsilon}^{-1},
\end{gathered}
$$

where $J$ is the Jacobian of the corresponding transformation which is given by

$$
J=\operatorname{det}\left(\frac{\partial(x-\epsilon)^{\mu}}{\partial x^{\nu}}\right) .
$$

Using all these relations, we can prove that the lagrangian density $\mathcal{L}_{0}$ is gravitational gauge covariant,

$$
\mathcal{L}_{0} \rightarrow \mathcal{L}_{0}^{\prime}=\hat{U}_{\epsilon} \mathcal{L}_{0} \hat{U}_{\epsilon}^{-1}
$$

Then eq.(3.22), eq.(3.23) and eq.(2.27) give out

$$
\mathcal{L} \rightarrow \mathcal{L}^{\prime}=J \cdot \hat{U}_{\epsilon} \mathcal{L} \hat{U}_{\epsilon}^{-1}
$$

Using the following relation,

$$
\int \mathrm{d}^{4} x J\left(\hat{U}_{\epsilon} f(x)\right)=\int \mathrm{d}^{4} x f(x),
$$

we can prove that the action of the system is gravitational gauge invariant,

$$
S \rightarrow S^{\prime}=S
$$

Therefore, the system has local gravitational gauge symmetry.

The action defined by eq.(2.31) has both local $U(1)$ gauge symmetry and local gravitational gauge symmetry. It means that it has local $G U(1)$ gauge symmetry. The $G U(1)$ gauge transformations of various fields and operators are

$$
\begin{gathered}
\psi \rightarrow \psi^{\prime}=(g(x) \psi), \\
C_{\mu} \rightarrow C_{\mu}^{\prime}=\hat{U}_{\epsilon} C_{\mu} \hat{U}_{\epsilon}^{-1}-\frac{1}{i g} \hat{U}_{\epsilon}\left(\partial_{\mu} \hat{U}_{\epsilon}^{-1}\right), \\
G_{\mu}^{\alpha} \rightarrow G_{\mu}^{\alpha}=\Lambda_{\beta}^{\alpha} g(x) G_{\mu}^{\beta} g^{-1}(x),
\end{gathered}
$$




$$
\begin{gathered}
G_{\alpha}^{-1 \mu} \rightarrow G_{\alpha}^{\prime-1 \mu}=\Lambda_{\alpha}^{\beta} g(x) G_{\beta}^{-1 \mu} g^{-1}(x), \\
A_{\mu} \rightarrow A_{\mu}^{\prime}=g(x)\left[A_{\mu}-\frac{1}{e}\left(D_{\mu} \alpha(x)\right)\right] g^{-1}(x), \\
\mathbb{D}_{\mu} \rightarrow \mathbb{D}_{\mu}^{\prime}=g(x) \mathbb{D}_{\mu} g^{-1}(x), \\
A_{\mu \nu} \rightarrow A_{\mu \nu}^{\prime}=g(x)\left[A_{\mu \nu}+\frac{g}{e} F_{\mu \nu}^{\sigma}\left(\partial_{\sigma} \alpha(x)\right)\right] g^{-1}(x), \\
F_{\mu \nu}^{\alpha} \rightarrow F_{\mu \nu}^{\prime \alpha}=\Lambda_{\beta}^{\alpha} g(x) F_{\mu \nu}^{\beta} g^{-1}(x), \\
\mathbb{A}_{\mu \nu} \rightarrow \mathbb{A}_{\mu \nu}^{\prime}=g(x) \mathbb{A}_{\mu \nu} g^{-1}(x), \\
J(C) \rightarrow J^{\prime}\left(C^{\prime}\right)=J \cdot g(x) J(C) g^{-1}(x),
\end{gathered}
$$

where $g(x)$ is given by eq.(2.5). The action eq.(2.31) is invariant under these transformations.

\section{Interactions}

Define

$$
\begin{aligned}
& A_{0 \mu \nu}=\partial_{\mu} A_{\nu}-\partial_{\nu} A_{\mu}, \\
& F_{0 \mu \nu}^{\alpha}=\partial_{\mu} C_{\nu}^{\alpha}-\partial_{\nu} C_{\mu}^{\alpha} .
\end{aligned}
$$

Then, we can separate the lagrangian density eq.(2.27) into free lagrangian density $\mathcal{L}_{F}$ and interaction lagrangian density $\mathcal{L}_{I}$,

$$
\mathcal{L}=\mathcal{L}_{F}+\mathcal{L}_{I}
$$

where,

$$
\mathcal{L}_{F}=-\frac{1}{4} \eta^{\mu \rho} \eta^{\nu \sigma} A_{0 \mu \nu} A_{0 \rho \sigma}-\bar{\psi}\left(\gamma^{\mu} \partial_{\mu}+m\right) \psi-\frac{1}{4} \eta^{\mu \rho} \eta^{\nu \sigma} \eta_{2 \alpha \beta} F_{0 \mu \nu}^{\alpha} F_{0 \rho \sigma}^{\beta},
$$




$$
\begin{aligned}
\mathcal{L}_{I}= & -\frac{1}{4} \eta^{\mu \rho} \eta^{\nu \sigma}\left(\sum_{n=1}^{\infty} \frac{1}{n !}\left(g \eta_{1 \alpha_{1}}^{\mu_{1}} C_{\mu_{1}}^{\alpha_{1}}\right)^{n}\right) A_{0 \mu \nu} A_{0 \rho \sigma} \\
& -\left(\sum_{n=1}^{\infty} \frac{1}{n !}\left(g \eta_{1 \alpha_{1}}^{\mu_{1}} C_{\mu_{1}}^{\alpha_{1}}\right)^{n}\right) \bar{\psi}\left(\gamma^{\mu} \partial_{\mu}+m\right) \psi \\
& -\frac{1}{4} \eta^{\mu \rho} \eta^{\nu \sigma} \eta_{2 \alpha \beta}\left(\sum_{n=1}^{\infty} \frac{1}{n !}\left(g \eta_{1 \alpha_{1}}^{\mu_{1}} C_{\mu_{1}}^{\alpha_{1}}\right)^{n}\right) F_{0 \mu \nu}^{\alpha} F_{0 \rho \sigma}^{\beta} \\
& +i e \cdot e^{I(C)} \bar{\psi} \gamma^{\mu} \psi A_{\mu}+g e^{I(C)} \bar{\psi} \gamma^{\mu}\left(\partial_{\alpha} \psi\right) C_{\mu}^{\alpha} \\
& +g e^{I(C)} \eta^{\mu \rho} \eta^{\nu \sigma}\left(\partial_{\mu} A_{\nu}-\partial_{\nu} A_{\mu}\right) C_{\rho}^{\alpha}\left(\partial_{\alpha} A_{\sigma}\right) \\
& -\frac{g}{2} e^{I(C)} \eta^{\mu \rho} \eta^{\nu \sigma} A_{\mu \nu} G_{\alpha}^{-1 \lambda} A_{\lambda} F_{\rho \sigma}^{\alpha} \\
& -\frac{g^{2}}{4} e^{I(C)} \eta^{\mu \rho} \eta^{\nu \sigma} G_{\alpha}^{-1 \kappa} G_{\beta}^{-1 \lambda} A_{\kappa} A_{\lambda} F_{\mu \nu}^{\alpha} F_{\rho \sigma}^{\beta} \\
& -\frac{g^{2}}{2} e^{I(C)} \eta^{\mu \rho} \eta^{\nu \sigma}\left(C_{\mu}^{\alpha} C_{\rho}^{\beta}\left(\partial_{\alpha} A_{\nu}\right)\left(\partial_{\beta} A_{\sigma}\right)-C_{\nu}^{\alpha} C_{\rho}^{\beta}\left(\partial_{\alpha} A_{\mu}\right)\left(\partial_{\beta} A_{\sigma}\right)\right) \\
& +g e^{I(C)} \eta^{\mu \rho} \eta^{\nu \sigma} \eta_{2 \alpha \beta}\left(\partial_{\mu} C_{\nu}^{\alpha}-\partial_{\nu} C_{\mu}^{\alpha}\right) C_{\rho}^{\delta}\left(\partial_{\delta} C_{\sigma}^{\beta}\right) \\
& -\frac{1}{2} g^{2} e^{I(C)} \eta^{\mu \rho} \eta^{\nu \sigma} \eta_{2 \alpha \beta}\left(C_{\mu}^{\delta} \partial_{\delta} C_{\nu}^{\alpha}-C_{\nu}^{\delta} \partial_{\delta} C_{\mu}^{\alpha}\right) C_{\rho}^{\epsilon}\left(\partial_{\epsilon} C_{\sigma}^{\beta}\right) .
\end{aligned}
$$

From eq.(4.4), we can write out propagators of gravitational gauge field, electromagnetic field and Dirac field. From the interaction lagrangian $\mathcal{L}_{I}$, we can see that Dirac field, electromagnetic field and gravitational gauge field couple each other. From eq.(4.5), we can write out Feynman Rules for various interaction vertices which is useful for calculation of Feynman diagrams.

\section{Equations of Motion and Conserved Currents}

The Euler-Lagrangian equation of motion of Dirac field is

$$
\left[\gamma^{\mu}\left(\partial_{\mu}-i e A_{\mu}-g C_{\mu}^{\alpha} \partial_{\alpha}\right)+m\right] \psi=0
$$

The equation of motion of electromagnetic field is

$$
\begin{aligned}
\partial^{\mu} \mathbb{A}_{\mu \nu}= & -i e \bar{\psi} \gamma_{\nu} \psi+g \eta^{\lambda \rho} \partial_{\mu}\left(C_{\lambda}^{\mu} \mathbb{A}_{\rho \nu}\right) \\
& -g \eta^{\kappa \rho} G_{\kappa}^{\mu} \mathbb{A}_{\rho \nu} \partial_{\mu}\left(\eta_{1 \alpha}^{\lambda} C_{\lambda}^{\alpha}\right)+\frac{g}{2} \eta^{\mu_{1} \rho} \eta^{\nu_{1} \sigma} \eta_{\nu \lambda} G_{\alpha}^{-1 \lambda} F_{\mu_{1} \nu_{1}}^{\alpha} \mathbb{A}_{\rho \sigma} .
\end{aligned}
$$

The equation of motion of gravitational gauge field is

$$
\partial^{\mu}\left(\eta^{\nu \sigma} \eta_{2 \alpha \beta} F_{\mu \sigma}^{\beta}\right)=-g T_{g \alpha}^{\nu},
$$


where $T_{g \beta}^{\sigma}$ is the gravitational energy-momentum tensor, whose explicit expression is

$$
\begin{aligned}
T_{g \alpha}^{\nu}= & \bar{\psi} \gamma^{\nu} \partial_{\alpha} \psi-\eta^{\mu_{1} \rho} \eta^{\nu \sigma}\left(\partial_{\alpha} A_{\mu_{1}}\right) \mathbb{A}_{\rho \sigma}-\eta^{\mu_{1} \rho} \eta^{\nu \sigma} \eta_{2 \alpha_{1} \beta} F_{\rho \sigma}^{\beta}\left(\partial_{\alpha} C_{\mu_{1}}^{\alpha_{1}}\right)+\eta_{1 \alpha}^{\nu} \mathcal{L}_{0} \\
& -\eta^{\mu_{1} \rho} \eta^{\nu \sigma} \eta_{2 \alpha \beta}\left(\partial_{\mu}\left(C_{\mu_{1}}^{\mu} F_{\rho \sigma}^{\beta}\right)\right)+\eta^{\mu_{1} \rho} \eta^{\nu \sigma} \partial_{\mu}\left(G_{\alpha}^{-1 \lambda} G_{\mu_{1}}^{\mu} A_{\lambda} \mathbb{A}_{\rho \sigma}\right) \\
& +\eta^{\mu \rho} \eta^{\nu \sigma} \eta_{2 \alpha \beta}\left(D_{\mu}\left(\eta_{1 \alpha_{1}}^{\lambda} C_{\lambda}^{\alpha_{1}}\right)\right) F_{\rho \sigma}^{\beta}-\frac{g}{2} \eta^{\mu_{1} \rho} \eta^{\nu_{1} \sigma} G_{\alpha_{1}}^{-1 \nu} G_{\alpha}^{-1 \lambda} A_{\lambda} \mathbb{A}_{\rho \sigma} F_{\mu_{1} \nu_{1}}^{\alpha_{1}} \\
& -g \eta^{\mu_{1} \rho} \eta^{\nu \sigma} G_{\alpha_{1}}^{-1 \lambda} A_{\lambda} \mathbb{A}_{\rho \sigma}\left(\partial_{\alpha} C_{\mu_{1}}^{\alpha_{1}}\right)+g \eta^{\mu \rho} \eta^{\nu \sigma}\left(D_{\mu}\left(\eta_{1 \alpha_{1}}^{\lambda_{1}} C_{\lambda_{1}}^{\alpha_{1}}\right)\right) G_{\alpha}^{-1 \lambda} A_{\lambda} \mathbb{A}_{\rho \sigma} .
\end{aligned}
$$

The global gravitational gauge symmetry gives out inertial energy-momentum tensor $T_{i \alpha}^{\mu}$,

$$
\begin{aligned}
T_{i \alpha}^{\mu}= & e^{I(C)}\left[\bar{\psi} \gamma^{\nu} G_{\nu}^{\mu} \partial_{\alpha} \psi+\eta^{\mu_{1} \rho} \eta^{\nu \sigma} G_{\mu_{1}}^{\mu} \mathbb{A}_{\rho \sigma}\left(\partial_{\alpha} A_{\nu}\right)\right. \\
& +\eta^{\mu \rho} \eta^{\nu \sigma} \eta_{2 \beta \gamma} F_{\rho \sigma}^{\gamma}\left(\partial_{\alpha} C_{\nu}^{\beta}\right)-g \eta^{\mu_{1} \rho} \eta^{\nu \sigma} \eta_{2 \beta \gamma} C_{\mu_{1}}^{\mu} F_{\rho \sigma}^{\gamma}\left(\partial_{\alpha} C_{\nu}^{\beta}\right) \\
& \left.+g \eta^{\mu_{1} \rho} \eta^{\nu \sigma} G_{\beta}^{-1 \lambda} G_{\mu_{1}}^{\mu} A_{\lambda} \mathbb{A}_{\rho \sigma}\left(\partial_{\alpha} C_{\nu}^{\beta}\right)+\delta_{\alpha}^{\mu} \mathcal{L}_{0}\right] .
\end{aligned}
$$

Compare eq.(5.4) with eq.(5.5), we can easy find that gravitational energy-momentum tensor is not equivalent to the inertial energy-momentum tensor. But when gravitational gauge field vanishes, they are equivalent. In this case, they are

$$
T_{i \alpha}^{\mu}=T_{g \alpha}^{\mu}=\bar{\psi} \gamma^{\mu} \partial_{\alpha} \psi+\eta^{\mu \rho} \eta^{\nu \sigma} A_{\rho \sigma}\left(\partial_{\alpha} A_{\nu}\right)+\delta_{\alpha}^{\mu} \mathcal{L}_{0}
$$

which is what we expected in traditional quantum field theory.

If we denote

$$
\begin{aligned}
\mathbb{J}_{\nu}= & i \bar{\psi} \gamma_{\nu} \psi-\frac{g}{e} \eta^{\lambda \rho} \partial_{\mu}\left(C_{\lambda}^{\mu} \mathbb{A}_{\rho \nu}\right)+\frac{g}{e} \eta^{\kappa \rho} G_{\kappa}^{\mu} \mathbb{A}_{\rho \nu} \partial_{\mu}\left(\eta_{1 \alpha}^{\lambda} C_{\lambda}^{\alpha}\right) \\
& -\frac{g}{2 e} \eta^{\mu_{1} \rho} \eta^{\nu_{1} \sigma} \eta_{\nu \lambda} G_{\alpha}^{-1 \lambda} F_{\mu_{1} \nu_{1}}^{\alpha} \mathbb{A}_{\rho \sigma} .
\end{aligned}
$$

Then, eq.(5.2) can be changed into

$$
\partial^{\mu} \mathbb{A}_{\mu \nu}=-e_{d_{\nu}} .
$$

Because,

$$
\partial^{\nu} \partial^{\mu} \mathbb{A}_{\mu \nu}=0,
$$

$\mathbb{J}_{\nu}$ is a conserved current,

$$
\partial^{\nu} \mathbb{J}_{\nu}=0
$$


It means that $\bar{\psi} \gamma_{\nu} \psi$ is no longer a conserve current,

$$
\partial^{\nu}\left(\bar{\psi} \gamma_{\nu} \psi\right) \neq 0
$$

Both gravitational field $C_{\mu}^{\alpha}$ and electromagnetic field $A_{\mu}$ contribute some to generalized electromagnetic current. In other words, in the unified theory, electromagnetic field is also a source of itself, which originates from the non-Able nature of the semidirect product group $G U(1)$. But if gravitational field vanishes, the electromagnetic current $\mathbb{J}_{\mu}$ will return to

$$
\mathbb{J}_{\nu}=i \bar{\psi} \gamma_{\nu} \psi
$$

which is just the traditional electromagnetic current.

\section{Summary}

In this paper, gravitational interactions and electromagnetic interactions are unified in a semi-direct product group. Because generator of $U(1)$ group and generators of gravitational gauge group have different dimension, that is, generator of $U(1)$ group are dimensionless while generators of gravitational gauge group have negative mass dimension, it is hard to unify $U(1)$ gauge interactions and gravitational gauge interactions in a simple group. Because of the difference of dimensions of generators, we need at least two independent parameters for coupling constant in any kind of unified theory. When we unify $U(1)$ gauge interactions and gravitational gauge interactions in $G U(1)$ group, we only need two independent parameters for coupling constant, this unified theory can be regarded as a kind of minimal theory of unification. It is impossible to unify gravitational interactions and electromagnetic interactions in a simple group in which only one independent coupling constant is used.

Because $U(1)$ gauge group and gravitational gauge group are unified in a semidirect product group, not in a direct product group, field strength of gravitational gauge field joins into the definition of gauge covariant field strength of $U(1)$ gauge field. This will cause additional interactions between $U(1)$ gauge fields and gravitational gauge field and affect the definition of generalized electromagnetic current.

\section{References}

[1] H.Weyl, in Raum. Zeit. Materia, 3rd ed. (Springer-Verlag, Berlin, 1920) 
[2] H.Weyl, Gravitation and Elektrizitat. Sitzungsber. Akademie der Wissenschaften Berlin, 465-480(1918). Siehe auch die Gesammelten Abhandlungen. 6 Vols. Ed. K.Chadrasekharan, Springer-Verlag.

[3] V.Fock, Zeit. f. Physik, 39 (1927) 226.

[4] H.Weyl, Zeit. f. Physik, 56 (1929) 330.

[5] W.Pauli, Handbuch der physik, Geiger and scheel, 2, Aufl., Vol. 24, Teil 1 pp. 83-272.

[6] R.Utiyama, Phys.Rev.101 (1956) 1597.

[7] A.Brodsky, D.Ivanenko and G. Sokolik, JETPH 41 (1961)1307; Acta Phys.Hung. 14 (1962) 21.

[8] T.W.Kibble, J.Math.Phys. 2 (1961) 212.

[9] D.Ivanenko and G.Sardanashvily, Phys.Rep. 94 (1983) 1.

[10] F.W.Hehl, J.D.McCrea, E.W.Mielke and Y.Ne'eman Phys.Rep. 258 (1995) 1171.

[11] F.W.Hehl, P. Von Der Heyde, G.D.Kerlick, J.M.Nester Rev.Mod.Phys. 48 (1976) 393-416.

[12] F.Gronwald and F.W.Hehl, On the Gauge Aspects of Gravity, gr-qc/9602013.

[13] F.Brandt, Phys.Rev. D64 (2001) 065025.

[14] Rolando Gaitan D., A possible gauge formulation for gravity?, gr-qc/0109079.

[15] M.Botta Cantcheef, General Relativity as a (constrained) Yang-Mills's theory, gr-qc/0010080.

[16] Ning Wu, Gauge Theory of Gravity, talk given at Meeting of the Devision of Particles and Fields of American Physical Society at the College of William \& Mery(DPF2002), May 24-28, 2002, Williamsburg, Virgia, USA; hepth/0109145.

[17] Ning Wu, Commun.Theor.Phys., 38 (2002): 151-156. 